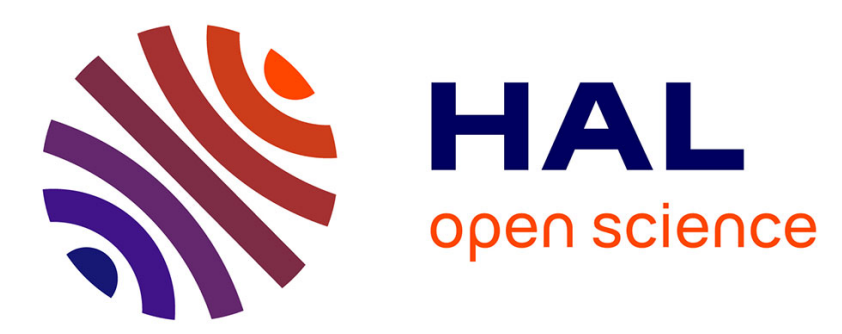

\title{
Atypical Effect of Displacement Damage on LM124 Bipolar Integrated Circuits
}

Thomas Borel, F. Roig, Alain Michez, B. Azais, S. Danzeca, N.J.-H. Roche, F. Bezerra, P. Calvel, L. Dusseau

\section{- To cite this version:}

Thomas Borel, F. Roig, Alain Michez, B. Azais, S. Danzeca, et al.. Atypical Effect of Displacement Damage on LM124 Bipolar Integrated Circuits. 54th Annual IEEE International Nuclear and Space Radiation Effects Conference, Jul 2017, New Orleans, United States. pp.71-77, 10.1109/TNS.2017.2772901 . hal-01781910

\section{HAL Id: hal-01781910 https://hal.science/hal-01781910}

Submitted on 21 Aug 2020

HAL is a multi-disciplinary open access archive for the deposit and dissemination of scientific research documents, whether they are published or not. The documents may come from teaching and research institutions in France or abroad, or from public or private research centers.
L'archive ouverte pluridisciplinaire HAL, est destinée au dépôt et à la diffusion de documents scientifiques de niveau recherche, publiés ou non, émanant des établissements d'enseignement et de recherche français ou étrangers, des laboratoires publics ou privés. 


\title{
Atypical Effect of Displacement Damage on LM124 Bipolar Integrated Circuits
}

\author{
T. Borel ${ }^{\circledR}$, F. Roig, A. Michez ${ }^{\circledR}$, B. Azais, S. Danzeca, N. J.-H. Roche ${ }^{\circledR}$, F. Bezerra, \\ P. Calvel, and L. Dusseau
}

\begin{abstract}
LM124 operational amplifiers from three different manufacturers are irradiated with ${ }^{60} \mathrm{Co}$ gamma rays and neutrons. During neutrons irradiation, one of the three integrated circuits exhibits an unexpected slew rates increase while its open loop gain and supply bias current follow the usual monotonic decrease as described in the literature. Analysis at circuit level shows that this phenomenon is due to an increase in the radiationinduced base current of the transistor used as buffer stage in the amplification chain. It is then demonstrated that a slight modification of the buffer transistor design, which is not implemented on the two other devices, enhances this phenomenon. Finally, the impact of the buffer transistor design on displacement damage and total ionizing dose response is investigated.
\end{abstract}

Index Terms-Analog circuits, bipolar integrated circuits (ICs), ionizing effect, total dose effect, total nonionizing dose.

\section{INTRODUCTION}

$\mathbf{T}$ HIS paper aims at showing how a physical mechanism, i.e., the displacement damage (DD)-induced degradation at transistor level in an integrated circuit (IC), may cause an unexpected response at circuit level and that this effect may be amplified due to a slight modification in the design. This paper refers to a DD test campaign led on LM124 operational amplifiers from three different manufacturers.

Electronics in spacecraft missions are subject to cumulative effects such as the total ionizing dose (TID) and the DD. These effects will alter the electrical and functional parameters of

T. Borel is with the Institut d'Electronique et des Systèmes, Université de Montpellier, 34095 Montpellier, France, and also with the European Organization for Nuclear Research, CH-1211 Geneva, Switzerland (e-mail: thomas.borel@cern.ch).

F. Roig is with Nuclétudes, F-91978 Courtaboeuf, France (e-mail: froig@nucletudes.com).

A. Michez and L. Dusseau are with the Institut d'Electronique et des Systèmes, Université de Montpellier, F-34095 Montpellier, France.

B. Azais is with the Direction Générale de l'Armement, F-75015 Paris, France.

S. Danzeca is with the European Organization for Nuclear Research, CH-1211 Geneva, Switzerland.

N. J.-H. Roche is with the Center Spatial Universitaire, Universite de Montpellier, F-34095 Montpellier, France.

F. Bezerra is with the Center National d'Etude Spatiales, F-31400 Toulouse, France.

P. Calvel is with Thales Alenia Space, F-31100 Toulouse, France.

Color versions of one or more of the figures in this paper are available online at http://ieeexplore.ieee.org. the components [1]. Usually, the parameters observed during radiation testing are the positive $\left(I_{\mathrm{CCP}}\right)$ and negative supply bias current $\left(I_{\mathrm{CCN}}\right)$, the open loop gain $(\mathrm{OLG})$, the slew rate (SR), the input bias currents ( $I_{\mathrm{INP}}$ and $\left.I_{\mathrm{INM}}\right)$, the input offset voltage $\left(V_{\mathrm{OS}}\right)$, and the input offset current $\left(I_{\mathrm{OS}}\right)$, which are all sensitive to both TID and DD. In many cases, the observed response is not only driven by the degradation of single transistors, but also by circuit effects consisting in the compensation or the enhancement of the degradation of a stage/transistor by the degradation of another stage/transistor. Dusseau et al. [1] and Barnaby et al. [2] have extensively studied circuit effects in linear devices and comparator devices. Roig et al. [3]-[5] highlighted the influence of circuit design modifications on LM124s from three different manufacturers. Indeed, modifications such as an increased value of the current biasing the amplification stage or the suppression of the current sources biasing the input common collector stages directly impact on the opamp radiation-induced degradation.

TID experiments on these three investigated opamps were previously carried out, reported in [3], [5], and [6], and analyzed mainly from the TID and design points of view. This paper focuses on DD results that lead to an unexpected rebound-like behavior of the SR degradation of one of the tested components. These results are investigated, and the root causes of this unusual phenomenon are presented in Sections III and IV.

Experimental details about the test campaigns are provided in Section II. Section III presents the results obtained highlighting the unexpected shape of the SR degradation curve of one of the LM124. The analysis performed and the hypothesis made to clarify the root causes of this phenomenon are presented in Section IV.

\section{EXPERIMENTAL DETAILS}

\section{A. Device Under Test}

The devices under test used during these irradiation campaigns are commercial off-the-shelf (COTS) LM124 opamps from three different manufacturers. In this paper, those components are labeled as LM124-A, LM124-B, and LM124-C. Each test was realized with eight components per manufacturer and the same manufacturer with the same date code.

All three components share the same internal electrical schematic, except for the values of the current sources, and for a slight difference in the input stage of the device LM124-C 
in which the current sources were removed. The latter having no effect on the SR degradation and impacting only the input stage of the component [3]. Therefore, this will not be discussed in this paper.

\section{B. Total Ionizing Dose Irradiation}

TID experiments were performed at TRAD (Toulouse) using ${ }^{60} \mathrm{Co}$ gamma rays source. The dose rate was $10 \operatorname{mrad}_{(\mathrm{SiO} 2)} \cdot \mathrm{s}^{-1}$, and the total dose delivered was $50 \mathrm{krad}_{(\mathrm{SiO} 2)}$. The parts were irradiated in two different configurations, all pins grounded (GND) and noninverter with gain of 11. In this abstract, only the GND configuration is presented.

It is generally assumed that the irradiation with all pin GND is considered as the worst case, but as shown in [7]-[9], this is not always the case. Velo et al. [9] have shown the impact of the bias configuration by running test on a COTS LM124 operational amplifier. Those tests highlighted the fact that not all of the parameters of a linear device see their sensitivity increase when GND. Indeed, TID tests have shown that GND components see their input bias current sensitivity decrease. However, in our case, only the $I_{\mathrm{CCP}}$, SR, and OLG are studied, so the GND configuration is considered as worst case for these parameters.

\section{Displacement Damage}

DD experiments were performed at the PROSPERO fission reactor (CEA, Valduc, France) using $1-\mathrm{MeV}$ equivalent neutrons. Two fluences were investigated, $1 \times 10^{12}$ and $5 \times 10^{12} \mathrm{n} \cdot \mathrm{cm}^{-2}$ (maximum cumulated level). The parts were irradiated in GND configuration.

\section{EXPERIMENTAL Results}

TID and DD results have previously been published and discussed in [3] and [5] and used to assess the effect of design modifications on the radiation-induced response. However, a specific effect observed was not explained and deserved a standalone paper.

Fig. 1 shows the degradation of $I_{\mathrm{CCP}}, \mathrm{SR}$, and $\mathrm{OLG}$ observed during ${ }^{60} \mathrm{Co}$ experiments. It represents the average value of electrical parameters obtained with eight samples per manufacturer in GND configuration. The error bars represent the minimum and maximum values obtained during different tests. As presented in Fig. 1, the LM124-B shows a quasiinsensitivity against TID. Indeed, none of its parameters strongly degrade. A variation of $41.6 \mu \mathrm{A}$ over $922.7 \mu \mathrm{V}$ is observed for $I_{\mathrm{CCP}}(4.51 \%)$ after $50 \mathrm{krad}_{(\mathrm{SiO} 2)}$. The SR itself degrades by $8.8 \%$ and the OLG by $5.5 \%$.

In comparison to the other manufacturers, the LM124-A shows a relatively low degradation of $46.5 \%$ for the $I_{\mathrm{CCP}}$, $91 \%$ for the SR, and $92 \%$ for the OLG. Regarding the LM124-C, a degradation of $60 \%$ of $I_{\mathrm{CCP}}$ is observed, $60 \%$ for the SR, and $99.9 \%$ for the GBO.

All of the manufacturer's parts show a strong degradation, but the SR and the OLG follow the same trend of degradation as the $I_{\mathrm{CCP}}$.

Fig. 2 shows the degradation of $I_{\mathrm{CPP}}, \mathrm{SR}$, and OLG observed during the $1-\mathrm{MeV}$ equivalent neutron experiments.
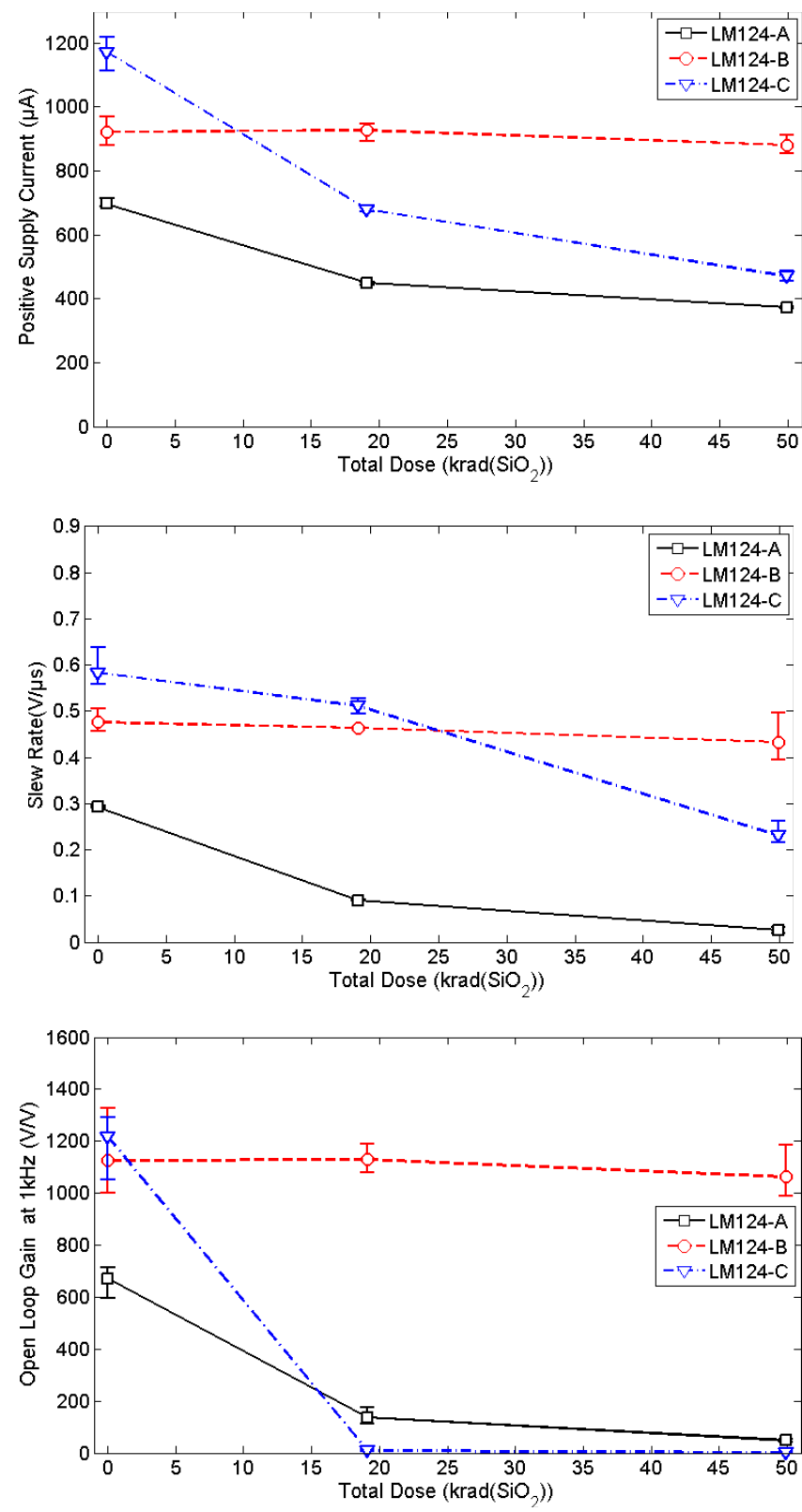

Fig. 1. Degradation of three LM124 operational amplifiers from different manufacturers (LM124-A, LM124-B, and LM124-C) irradiated with ${ }^{60} \mathrm{Co}$ in GND configuration [1]. The first curve represents the degradation of the current consumption as function as the TID. The second curve represents degradation of the SR as function as the TID. The third curve represents the degradation of the OLG as function as the TID. The dose rate is $10 \mathrm{mrad}_{(\mathrm{SiO} 2)} \cdot \mathrm{s}^{-1}$.

They present the average value of electrical parameters obtained with eight samples per manufacturer in GND configuration. The error bars represent the minimum and maximum values obtained during different tests. The LM124-A and LM124-B show a similar degradation on both parameters whereas the LM124-C shows a larger degradation. Indeed, for a dose of $5 \times 10^{12} \mathrm{n} \cdot \mathrm{cm}^{-2}$ a variation of $\Delta I_{\mathrm{CPP}}$ for $146 \mu \mathrm{A}(22.3 \%)$ and $155 \mu \mathrm{A}(25.7 \%)$, respectively, for LM124-A and LM124-B was observed. However, for LM124-C, a degradation of $378 \mu \mathrm{A}(33 \%)$ for the $\Delta I_{\mathrm{CPP}}$ was observed. Regarding the OLG, the trend of degradation is similar for the LM124-A and for the LM124-B, 

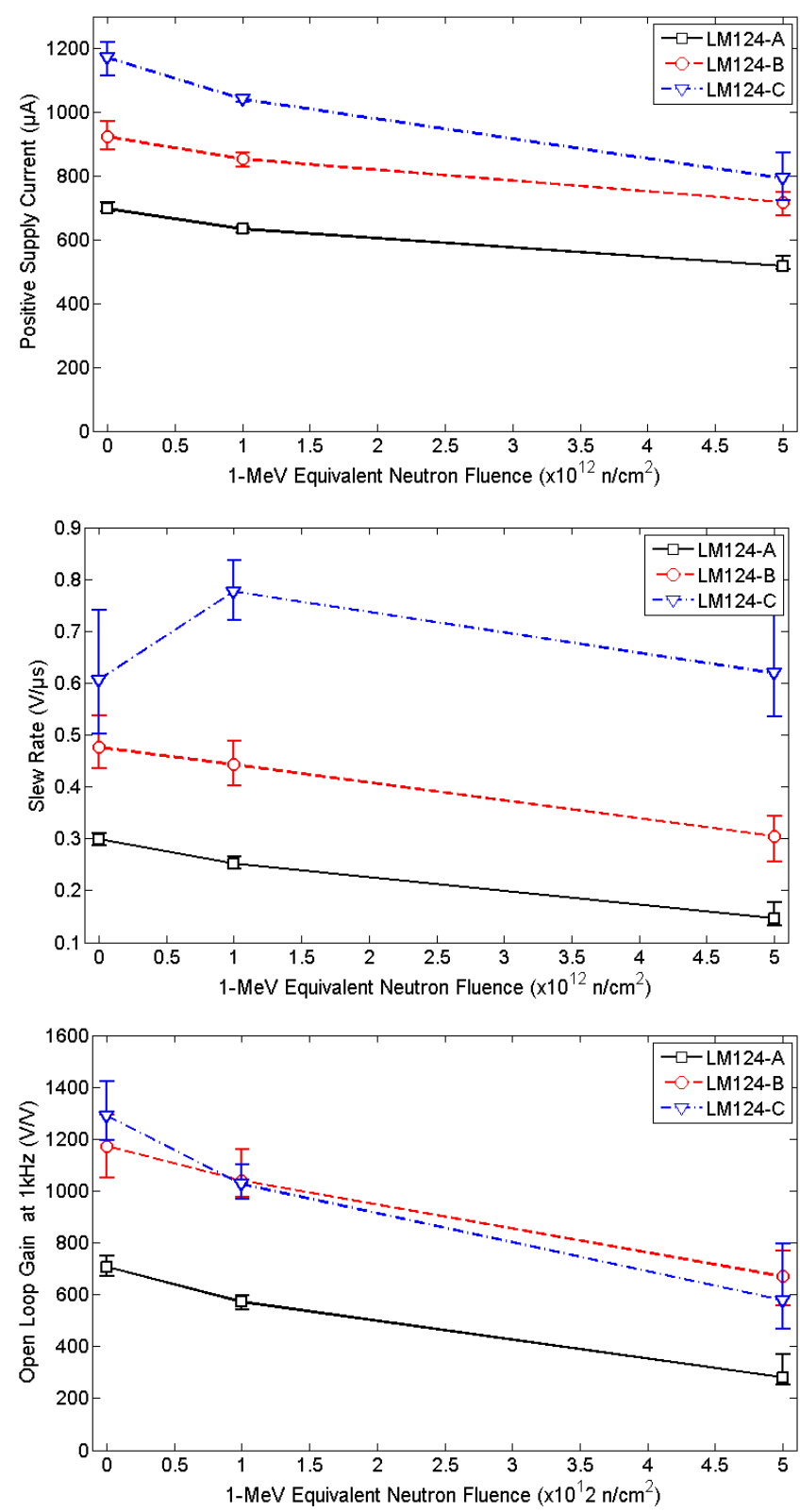

Fig. 2. Degradation of three LM124 operational amplifiers from different manufacturers (LM124-A, LM124-B, and LM124-C) irradiated with 1-MeV neutron equivalent in GND configuration [6]. The first curve represents the degradation of the current consumption as function as the DD. The second curve represents degradation of the SR as function as the DD. The third curve represents the degradation of the OLG as function as the DD.

whereas the LM124-C shows a more significant degradation trend. Concerning the SR, we still observe the same trend of degradation for the LM124-A and for the LM124-B; however, the LM124-C shows a different degradation.

As shown in [1] and [5], current in current sources decreases when irradiated with either TID or DD. This well-known mechanism is typical from the radiation-induced degradation of a current mirror [1]. On devices LM124-A and LM124-B, the SR, which is proportional to the value of the current sources, degrades following the same trend as the supply bias current. However, on the curves of Fig. 2 the SR of the LM124-C device exhibits an unusual degradation shape. Not only does the degradation show no decrease up

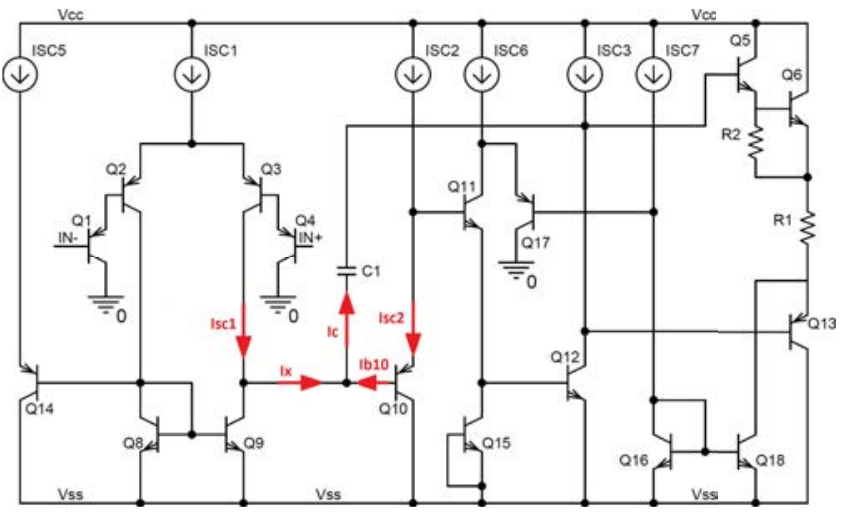

Fig. 3. Simplified internal electrical schematic of the LM124.

to $5 \times 10^{12} \mathrm{n} \cdot \mathrm{cm}^{-2}$, but it also increases significantly for a $1 \times 10^{12} \mathrm{n} \cdot \mathrm{cm}^{-2}$ fluence.

That behavior suggests that an additional internal mechanism is at play for the DD degradation in this specific component. In order to determine where this phenomenon happens on the LM124-C and what additional mechanism is at play, an analysis at circuit level was performed as presented in Section IV.

\section{Analysis at Circuit Level}

Fig. 3 presents the simplified electrical schematic of the three LM124s. This schematic can be decomposed into three different parts:

1) the differential part, composed of Q1-Q4, Q8, and Q10;

2) the amplification stage, composed of Q10-Q12 and Q15;

3) the output stage, composed of Q5, Q6, Q13, and Q16-Q18.

In this schematic, the currents sources named $I_{\mathrm{SC} 1}$ to $I_{\mathrm{SC} 3}$ and $I_{\mathrm{SC} 5}$ to $I_{\mathrm{SC} 7}$ are represented by the standard symbol of the current sources. Those currents are actually generated by three different transistors, two of them are spread collector transistors, and the other one is a single transistor.

In order to generate a specific current in the track, all of those transistors are all driven by the same base voltage. Different current values in the branches are obtained by varying the size of the collector area.

\section{A. Slew-Rate Mechanism}

The SR corresponds to the maximum slope of the output signal that can be achieved by the component. It is measured by applying a square wave on one of the input terminals and recording the slope of the output signal after the linear slope. It is expressed in $\mathrm{V} / \mu \mathrm{s}$ and is caused by the charge of the compensation capacitor, named $C_{1}$ in Fig. 3 , by the constant current delivered by $I_{\mathrm{SC} 1}$ and $I_{\mathrm{B} 10}$. In our case, the current $I_{\mathrm{B} 10}$ corresponds to the value of the current $I_{\mathrm{SC} 2}$ divided by the current gain $\beta_{10}$, and so, it can be neglected in comparison with the value of $I_{\mathrm{SC} 2}$ as long as the current gain of the transistor Q10 remains high enough.

The SR can be estimated using

$$
\mathrm{SR}=I_{C} / C_{1}
$$


where $I_{C}$ is the charge current and $C_{1}$ is the value of the compensation capacitor.

When the output switches from a high level output state to a low level output state, just before the process of commutation, the capacitor $C_{1}$ is empty. When the commutation starts, the capacitor begins to charge, with the current $I_{C}$ being equal to $I_{X}$ coming from the current source $I_{\mathrm{SC} 1}$.

Equation (1) demonstrates this during irradiation, as the current from the current sources decreases, the SR is bound to decrease [10]. Instead, on the LM124-C, the SR increases as observed in Fig. 2 until the fluence reaches $1 \times 10^{12} \mathrm{n} \cdot \mathrm{cm}^{-2}$, and then slightly decreases.

According to (1), this phenomenon may be caused either by the current which increases, or by a decrease in the capacitor value. Let us first examine the later hypothesis. As we know, the capacitor value is not supposed to decrease during the irradiation. However, before irradiation, we can observe on the curves Figs. 1 and 2 that the values of the $\mathrm{SR}$ of the pristine device $\mathrm{C}$ are higher than those of the SR of devices A and B. A first analysis has been completed and is presented in Section IV-A. Section IV-B presents the analysis carried out on the variation of the current value during the irradiation.

\section{B. Degradation Impact of the Transistor Q10}

The SR is directly linked to the current source named $I_{\mathrm{SC} 1}$ in Fig. 3 used to bias the differential stage. If $I_{\mathrm{SC} 1}$ decreases, the SR should decrease according to (1). In our case, we observe the opposite phenomenon. Instead of following the current source degradation which decreases, the SR increases. The only possible explanation for the observed increase in the SR is the current injected in $C_{1}$, which is no longer composed of $I_{\mathrm{SC} 2}$ only. Considering the node at $C_{1}$ input terminal, this current can only come from the base of Q10 which was previously neglected because of its low value on a pristine component.

This transistor is used to increase the amplification stage input impedance. In our case, Q10 is biased by the current source $I_{\mathrm{SC} 2}$. Some papers show that the radiation-induced current decrease of the current sources is not linear [2]. Therefore, the first degradation observed on Q10 is an increase of its base current. This increase adds a new contribution to the current charging the capacitor and thus increases the SR until $I_{\mathrm{SC} 2}$ itself degrades which will eventually decrease the SR.

Indeed, Roig et al. [3] showed that all parameters of the LM124-C (including SR and OLG) are twice that of LM124-A and 1.33 times higher than LM124-B. According to this, the current source $I_{\mathrm{SC} 2}$ should be greater for the LM124-C than for the other devices, and so, the base current of Q10 should also be more important for the LM124-C.

Another mechanism induced by DD may explain the strong enhancement of this current in device LM124-C. Fig. 4 shows a photomicrography of the transistors Q10 and Q14 of the LM124-C. As we can see, each transistor is nested within another transistor. Because of this specific structure, the transistor Q10 and Q14 are composed of more junction than a normal transistor. Rodriguez et al. [11] have shown that when

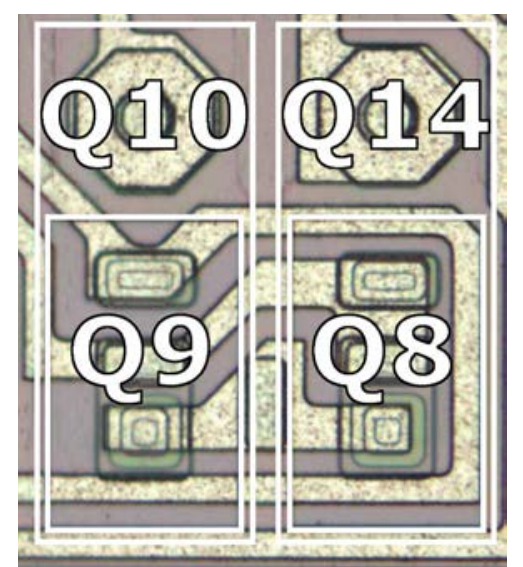

Fig. 4. Microphotography of the transistor Q10 in the LM124-C including Q9 inside its base region.

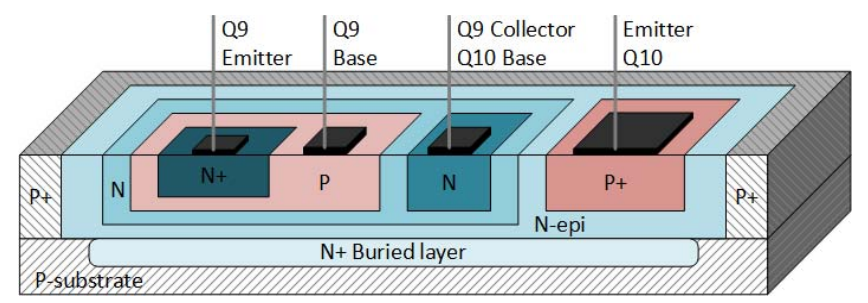

Fig. 5. Estimated cross section of the Q9 and Q10 structures.

a cluster of defect is created inside a depletion region, because of thermal generation, the cluster act as current generator. Thus, we suppose that depending on the location of the cluster, a significant increase of the Q10 base current can be induced by this thermal generation. This hypothesis can also explain the spread of the SR values in Fig. 2.

The estimated cross section is presented in Fig. 5. Q10 was designed with another transistor inside its base. Because of this design and because Q9 and Q10 are, respectively, a vertical NPN transistor and a substrate PNP transistor, the current passes vertically to the substrate. Barnaby et al. [2], [12] and Rax et al. [13] showed that the degradation of vertical NPN, vertical PNP, and substrate PNP transistors are more sensitive to proton and neutron irradiations. Those irradiations simultaneously degrade the surface recombination velocity, the net positive oxide charge and the collector-region lifetime (the base region of the two pnp transistors), which is a very sensitive parameter for bipolar technologies. As a result, both particles will subsequently cause an increased degradation of the current gain. Moreover, wider and deeper structures have an increased base width, which is the variable sensitive to DD.

Because of this specific structure, the neutron-induced degradation will reduce drastically the gain of the transistor Q10 and consequently the base current of this transistor will increase at the very beginning of the irradiation.

\section{Capacitor Value Before Irradiation}

Considering the values of the SR before irradiation shown in Fig. 2, the SR is already higher for the LM124-C than the other components. According to (1), either the current source value is higher on the LM124-C, or the capacitor value is smaller. 


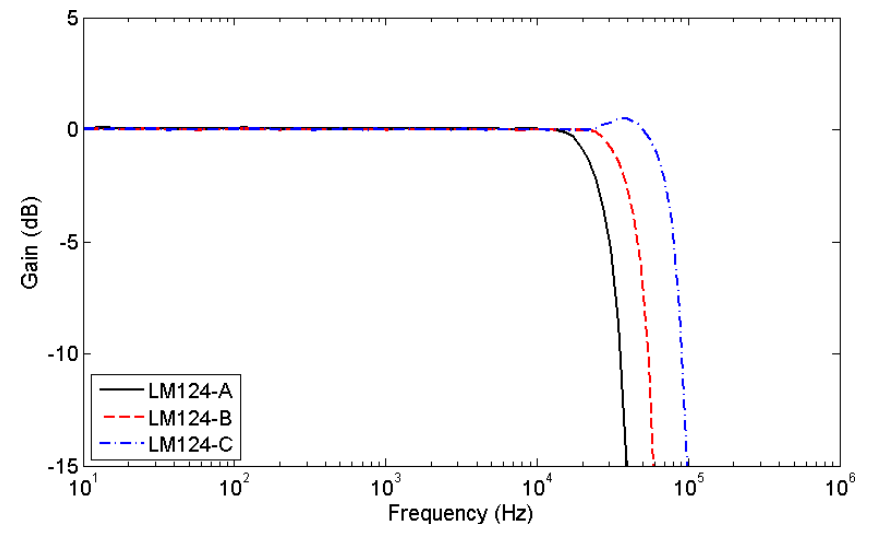

Fig. 6. Results of the frequency analysis done on three different LM124s.

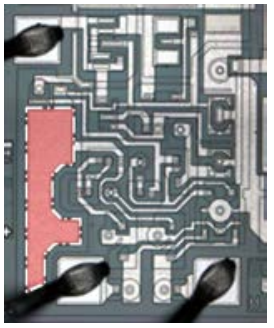

(a)

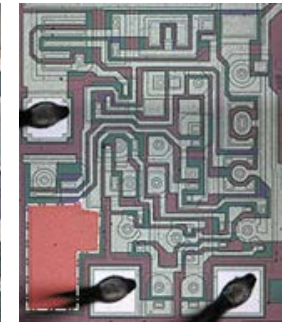

(b)

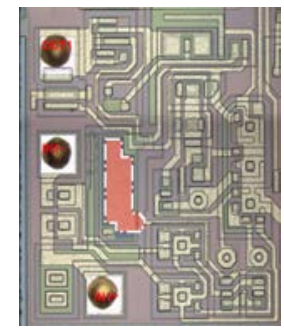

(c)
Fig. 7. Photomicrography of one-quarter of each of the three dies. These photographs show the capacitor size depending of the chip. (a) LM124-A, (b) LM124-B, and (c) LM124-C. In these photographs, the capacitor is highlighted in red and surrounded with white dashes.

In order to estimate the relative value of the capacitor $C_{1}$ between the three components, a frequency analysis was conducted and the results are presented in Fig. 6. Each curve presents the average value obtained with ten samples per manufacturer. As we can observe, the LM124-C shows the highest cutoff frequency. The LM124-A shows a cutoff frequency of $2.6 \times 10^{4}$ and $4.04 \times 10^{4} \mathrm{~Hz}$ for the LM124-B, and $7.26 \times 10^{4} \mathrm{~Hz}$ for LM124-C. This is remarkable since the LM124C also exhibits the highest OLG which should impact on the Miller effect and reduce the cutoff frequency instead of increasing it.

According to these results, we can consider that the value of the capacitor $C_{1}$ of the LM124-C is smaller than for the other manufacturers' parts.

In order to get another hint on the capacitor value, the size of the capacitor on the chip was investigated visually. Fig. 7 presents the photomicrography of different dies for each manufacturer with, highlighted, the location of the capacitor on the die.

It is understand that the visual size of the capacitor is not the only parameter conditioning the capacitor's value since the oxide thickness and the oxide permittivity impact on this value. Nevertheless, it is reasonable to think that a smaller size is an indication of a smaller value.

As we can see, the size of the capacitor for the LM124-C seems to be the smallest of the three components. In order to estimate the real size of this capacitor, the area on the chip was compared to the total area of the chip.

Table I summarizes the estimated size of each capacitor on the chip. Capacitor $C_{1}$ is much smaller compared to the
TABLE I

ESTIMATED Size of THE CAPACITOR DEPENDING ON THEIR SIZE ON THE CHIP

\begin{tabular}{c|cc|cc|cc}
\hline \hline Component & \multicolumn{2}{|c|}{ LM124-A } & \multicolumn{2}{c|}{ LM124-B } & \multicolumn{2}{c}{ LM124-C } \\
\hline Parameter & Area & Area & Area & Area & Area & $\begin{array}{c}\text { Area } \\
(\%)\end{array}$ \\
& $(\mu \mathrm{m})$ & $(\%)$ & $(\mu \mathrm{m})$ & $(\%)$ & $(\mu \mathrm{m})$ \\
\hline Chip & 100 & 182.09 & 100 & 215.84 & 100 & 183.48 \\
Capacitor & 3.27 & 5.96 & 2.85 & 6.15 & 1.12 & 2.05 \\
\hline \hline
\end{tabular}

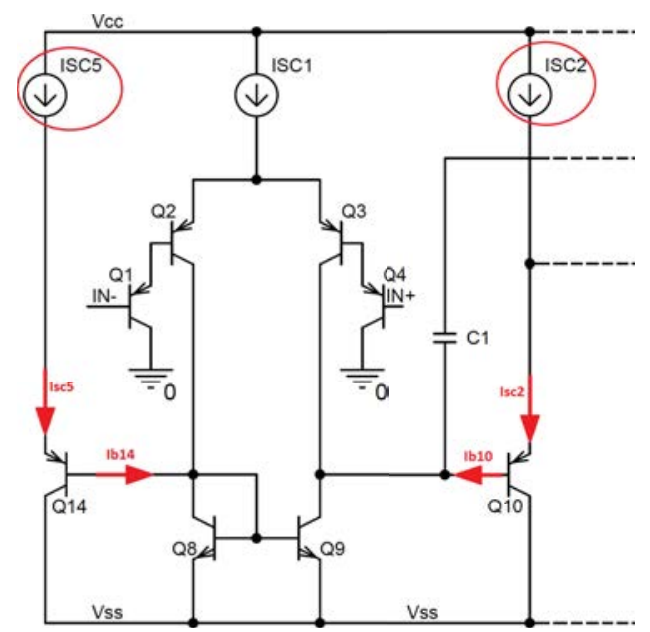

Fig. 8. Differential pair of the LM124 showing the current of the compensation structure formed by the transistors Q10 and Q14.

other manufacturers. For the LM124-A, the size occupied by the capacitor is $5.96 \mu \mathrm{m}^{2}$. A similar size was measured for the LM124-B, but for the LM124-C the occupied area on the chip is $2.05 \mu \mathrm{m}^{2}$, which corresponds to a size reduction of 34\% compared to the LM124-A and of 33\% compared to the LM124-B.

To conclude, all three investigations i.e., the frequency measurements, the impact of the miller effect with the higher OLG before irradiation and the size of the capacitor on the chip, suggest that the capacitor value of the LM124-C is much smaller than the others components.

According to (1), a smaller value of capacitor $C_{1}$ will increase the sensitivity of the SR. Indeed, a smaller capacitor will amplify the effect of an increase in the charge current on the SR.

\section{Compensation Structure Formed by Q10 and Q14}

The structure formed by the transistors Q10 and Q14 is used to compensate any variation of the differential pair of Q10 induced by the temperature or by its leakage current. As presented in Fig. 8, in order to ensure a perfect equilibrium of the differential pair, the transistors Q8 and Q9 must be the same to have the same amount of current driven by the current mirror and ensure a proper parallelism in the two branches. Therefore, the transistors Q10 and Q14 must have the same design.

In our case, when the transistors Q10 and Q14 degrade, if their current sources, known, respectively, as $I_{\mathrm{SC} 2}$ and $I_{\mathrm{SC} 5}$, are different, a disequilibrium will be observed for the differential pair, and consequently the SR will be impacted. 


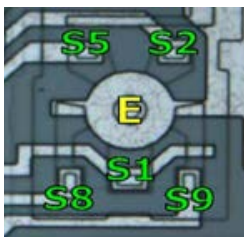

(a)

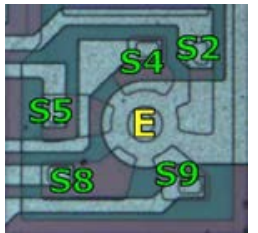

(b)

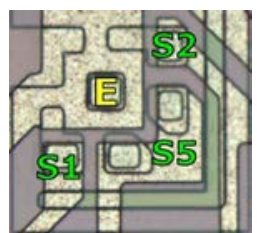

(c)
Fig. 9. Photomicrography of spread collector transistor of the three chips. (a) LM124-A, (b) LM124-B, and (c) LM124-C. Those transistors are used to provide the current for some parts of the circuit. On these photographs, the labels S1, S2, S5, S8, and S9 corresponds, respectively, to the current sources, $I_{\mathrm{SC} 1}, I_{\mathrm{SC} 2}, I_{\mathrm{SC} 5}, I_{\mathrm{SC} 8}$, and $I_{\mathrm{SC} 9}$. On the LM124-C, the current sources $I_{\mathrm{SC} 8}$ and $I_{\mathrm{SC} 9}$ were removed by the manufacturer.

TABLE II

Estimated Value of the CuRRent Sources DEPENDING ON THEIR SIZE

\begin{tabular}{c|cc|cc|cc}
\hline $\begin{array}{c}\text { Current } \\
\text { source }\end{array}$ & \multicolumn{2}{|c|}{ LM124-A } & \multicolumn{2}{c|}{ LM124-B } & \multicolumn{2}{c}{ LM124-C } \\
\hline Parameter & $\begin{array}{c}\text { Area } \\
(\%)\end{array}$ & $\begin{array}{c}\text { Estimated } \\
\text { current } \\
(\mu \mathrm{A})\end{array}$ & $\begin{array}{c}\text { Area } \\
(\%)\end{array}$ & $\begin{array}{c}\text { Estimated } \\
\text { current } \\
(\mu \mathrm{A})\end{array}$ & $\begin{array}{c}\text { Area } \\
(\%)\end{array}$ & $\begin{array}{c}\text { Estimated } \\
\text { current } \\
(\mu \mathrm{A})\end{array}$ \\
\hline S1 & 21.5 & 6.0 & 18.9 & 6.0 & 28.1 & 6.0 \\
S2 & 15.1 & 4.2 & 19.1 & 6.1 & 30.3 & 6.5 \\
S5 & 15.6 & 4.3 & 20.3 & 6.4 & 41.7 & 8.9 \\
S8 & 23.5 & 6.4 & 26.1 & 8.3 & - & - \\
S9 & 24.5 & 6.8 & 15.4 & 4.9 & - & - \\
\hline \hline
\end{tabular}

\section{E. Current Source Determination}

In order to have a good estimation of the current source values, an analysis of the spread collector transistor, used to provide the current for each part of the circuit, was performed.

Fig. 9 presents the spread collector transistor for each chip. Fig. 9(a)-(c) corresponds to the chip LM124-A, LM124-B, and LM124-C, respectively.

In order to create several different currents from a single transistor, the spread collector transistor is commonly used in analog devices. It can generate different currents values by shaping each collector area individually with different sizes. On this spread collector, only the current sources $I_{\mathrm{SC} 1}$, $I_{\mathrm{SC} 2}, I_{\mathrm{SC} 5}, I_{\mathrm{SC} 8}$, and $I_{\mathrm{SC} 9}$ are generated because they have a relatively close value. In comparison with $I_{\mathrm{SC} 3}, I_{\mathrm{SC} 6}$, and $I_{\mathrm{SC} 7}$ witch generate a higher current.

In order to estimate the value of the current for each current source on the same component, the collector zone was measured and compared to the size of the current source $I_{\mathrm{SC} 1}$ which was taken as reference with a value of $6 \mu \mathrm{A}$.

These values are not correlated with the real values of the current sources, but give an analogy with respect to the actual values of the current sources.

Table II presents the estimated values for each current source on the spread collector transistor. On the chip of the LM124-C, $I_{\mathrm{SC} 8}$ and $I_{\mathrm{SC} 9}$ were removed by design and are not present in Table II.

\section{F. Hypothesis Conclusion}

As we can see in Table II, the values of the LM124-C do not respect the working condition established in Sections IV-A-IV-E. Indeed, the point approached in Sections IV-A-IV-E presenting the hypothesis explaining the shape of the SR are all demonstrate true by the following results.

- When the transistor Q10 degrades, due to the current $I_{\mathrm{SC} 2}$ being bigger than $I_{\mathrm{SC} 1}$, the base current $I_{\mathrm{B} 10}$ adds to the current $I_{X}$ in Fig. 3 and is no longer negligible, affecting the SR directly.

- When the compensation structure formed by the transistors $\mathrm{Q} 10$ and $\mathrm{Q} 14$ degrades, due to $I_{\mathrm{SC} 2}$ and $I_{\mathrm{SC} 5}$ not being equal, this creates a disequilibrium in the differential pair, which affects the SR directly.

All of the points prove that atypical behavior observed with the LM124-C is mainly caused by the current sources.

\section{DisCUSSION}

System designers are sometimes constrained to use COTS for various reasons (cost and availability). As presented in this paper, screening of parts has to be done properly. Indeed, this paper points to the fact that a component with the same reference coming from different a manufacturer does not ensure the same behavior and reaction against radiation. Because each manufacturer has to design their own component with their own parameters involving the placement, the transistors types, the doping, etc., implies that each component will differ from the other components and will have to be tested in order to ensure system reliability against radiation.

Furthermore, parameters of linear operational amplifiers do not degrade in a monotonic way. Thereby, measurements during tests must take this non linearity into account, and intervals between measurements of parameters must be chosen to be sure that all phenomenon occurring during irradiation are highlighted. Indeed, in our case, without the step at $1 \times 10^{12} \mathrm{n} \cdot \mathrm{cm}^{-2}$ fluence, during the DD tests campaigns, no atypical behavior would have been detected, and moreover, depending on the specifications, this component would have displayed a perfect non degradation of the SR.

\section{CONCLUSION}

This paper addresses the questions raised by the unexpected increase of the SR observed during neutron irradiation on an LM124 whose design differs slightly from the other manufacturers.

It was shown that an increase in the base current of Q10 is responsible for the shape of the DD-induced degradation of the LM124-C. It was also shown that both the increased value of the current sources biasing the stages and the decreased value of the capacitor strongly enhance this effect which is not observed on devices with the usual design. Moreover, it was shown that the compensation structure created by Q10 and Q14, which is mainly designed in order to compensate the fluctuation in the differential pair, create a disequilibrium in the differential pair during the irradiation, which enhance the trend observer on the SR.

Finally, it was shown that the nested structure composed of Q9 and Q10 makes this stage more sensitive to DD than it is for TID.

Whatever the designer's motivation for tuning the current values and changing the design at transistor level, increasing the performance, TID hardening, etc., this result shows one more time that it is critical to screen parts properly and that 
devices with the same name from different manufacturers may exhibit very different behaviors.

\section{REFERENCES}

[1] L. Dusseau et al., "Review and analysis of the radiation-induced degradation observed for the input bias current of linear integrated circuits," IEEE Trans. Nucl. Sci., vol. 55, no. 6, pp. 3174-3181, Dec. 2008.

[2] H. J. Barnaby, R. D. Schrimpf, A. L. Sternberg, V. Berthe, C. R. Cirba, and R. L. Pease, "Proton radiation response mechanisms in bipolar analog circuits," IEEE Trans. Nucl. Sci., vol. 48, no. 6, pp. 2074-2080, Dec. 2001.

[3] F. Roig et al., "Modeling and investigations on TID-ASETs synergistic effect in LM124 operational amplifier from three different manufacturers," IEEE Trans. Nucl. Sci., vol. 60, no. 6, pp. 4430-4438, Dec. 2013.

[4] F. Roig et al., "Study of manufacturing variations impact on TID-ATREEs synergistic effect in LM124 operational amplifier," in Proc. 14th Eur. Conf. Radiation Effects Components Syst. (RADECS), Sep. 2013, pp. 1-4.

[5] F. Roig et al., "Impact of neutron-induced displacement damage on the ATREE response in LM124 operational amplifier," IEEE Trans. Nucl. Sci., vol. 61, no. 6, pp. 3043-3049, Dec. 2014.

[6] F. Roig et al., "Study and modeling of the impact of TID on the ATREE response in LM124 operational amplifier," IEEE Trans. Nucl. Sci., vol. 61, no. 4, pp. 1603-1610, Aug. 2014.
[7] K. Kruckmeyer, L. McGee, B. Brown, and D. Hughart, "Low dose rate test results of national semiconductor's ELDRS-free bipolar amplifier LM124 and comparators LM139 and LM193," in Proc. IEEE Radiation Effects Data Workshop, Jul. 2008, pp. 110-117.

[8] C. C. Yui, S. S. McClure, B. G. Rax, J. M. Lehman, T. D. Minto, and M. D. Wiedeman, "Total dose bias dependency and ELDRS effects in bipolar linear devices," in Proc. IEEE Radiation Effects Data Workshop, Jul. 2002, pp. 131-137.

[9] Y. G. Velo et al., "Bias effects on total dose-induced degradation of bipolar linear microcircuits for switched dose-rate irradiation," IEEE Trans. Nucl. Sci., vol. 57, no. 4, pp. 1950-1957, Aug. 2010.

[10] L. Dusseau et al., "Analysis of total-dose response of a bipolar voltage comparator combining radiation experiments and design data," IEEE Trans. Nucl. Sci., vol. 53, no. 4, pp. 1910-1916, Aug. 2006.

[11] A. Rodriguez et al., "Proton-induced single-event degradation in SDRAMs," IEEE Trans. Nucl. Sci., vol. 63, no. 4, pp. 2115-2121, Aug. 2016.

[12] H. J. Barnaby, S. K. Smith, R. D. Schrimpf, D. M. Fleetwood, and R. L. Pease, "Analytical model for proton radiation effects in bipolar devices," IEEE Trans. Nucl. Sci., vol. 49, no. 6, pp. 2643-2649, Dec. 2002.

[13] B. G. Rax, A. H. Johnston, and T. Miyahira, "Displacement damage in bipolar linear integrated circuits," IEEE Trans. Nucl. Sci., vol. 46, no. 6 , pp. 1660-1665, Dec. 1999. 